\title{
Erratum
}

\section{The Positivity of the Pressure in Thomas-Fermi Theory}

R. Benguria and E. H. Lieb

Commun. Math. Phys. 63, 193-218 (1978)

The conclusion of Lemma 2.4 should read:

then $\sum_{i=1}^{k} w_{i} f_{i}(\underline{z}+\lambda \underline{w})$ is increasing in $\lambda$ for $\lambda \geqq 0$ and $\underline{z}, \underline{w} \in \mathbb{R}_{+}^{k}$.

In the proof of Corollary 2.5 "...f $f_{i}$ is increasing" should be replaced by “... $\left.\Sigma_{2}\right)_{i} f_{i}\left(\lambda \underline{z}_{2}+z_{1}\right)$ is increasing ...". Lemma 2.4 was used only to prove Corollary 2.5 , and the latter was used in the equation following (4.42). 